\title{
Probabilistic prediction for the ampacity of overhead lines using Quantile Regression Neural Network
}

\author{
Xu Jin ${ }^{1}$, Fudong Cai ${ }^{2}$, Mengxia Wang ${ }^{1 *}$, Yang Sun $^{3}$ and Shengyuan Zhou ${ }^{1}$ \\ ${ }^{1}$ Key Laboratory of Power System Intelligent Dispatch and Control Ministry of Education, Shandong University, Jinan, Shandong, \\ 250061, China \\ ${ }^{2}$ Shandong Senter Electronic Co., Ltd., Zibo, Shandong, 255088, China \\ ${ }^{3}$ Zibo Vocational institute, Zibo, Shandong, 255314, China
}

\begin{abstract}
The ampacity of overhead transmission lines play a key role in power system planning and control. Due to the volatility of the meteorological elements, the ampacity of an overhead line is timevarying. In order to fully utilize the transfer capability of overhead transmission lines, it is necessary to provide system operators with accurate probabilistic prediction results of the ampacity. In this paper, a method based on the Quantile Regression Neural Network (QRNN) is proposed to improve the performance of the probabilistic prediction of the ampacity. The QRNN-based method uses a nonlinear model to comprehensively model the impacts of historical meteorological data and historical ampacity data on the ampacity at predictive time period. Numerical simulations based on the actual meteorological data around an overhead line verify the effectiveness of the proposed method.
\end{abstract}

\section{Introduction}

Nowadays, the rapid development of renewable energy power generation technology and constant increase of electricity consumption bring great challenges to the transfer capability of the power grid [1]. The shortage of transmission capacity will limit the accommodation of renewable energy and cause transmission congestion. In order to deal with these challenges, constructing new transmission lines may be a good choice, but it is timeconsuming and uneconomical [2]. For this reason, system operators should find effective strategies to increase the transmission capacity of existing power grid.

Conventionally, the ampacity of an overhead line is calculated under conservative weather assumptions, which is called static thermal rating (STR) [3]. Since conservative weather conditions are rare, the use of STR often causes under-utilization of the transmission capacity of an overhead line. Different from STR, the dynamic thermal rating (DTR) calculates the ampacity based on real-time measured meteorological data. The DTR technique can reveal the actual transmission capacity of an overhead line, and is usually higher than STR [4]. Thus, DTR is an effective tool to enhance the absorption of renewable energy and exploit the transfer capability of existing power grid [5].

For power system operators, knowing the ampacity ahead of time can help them make better control decisions and fully utilize the transfer capability of transmission lines. Therefore, many ampacity prediction methods have been developed based on the DTR technique. In [6] and [7], four meteorological elements around the overhead conductor were forecasted firstly, and then the prediction of the ampacity was obtained based on the forecasted meteorological data and heat balance equation. These two studies all focused on the determined prediction of the ampacity, which only provided the expected value of the ampacity at predictive time period. As a result, determined prediction methods are unable to provide the uncertainty information of the ampacity prediction results. In this case, probabilistic prediction methods of the ampacity were developed. In [8], a statistical method was proposed to forecast the probability density function (PDF) of the ampacity based on the numerical weather predictions (NWPs). In [9], historical meteorological data and NWPs were taken as the input of the expectation maximization algorithm to forecast the PDFs of meteorological elements. Then, based on the forecasted PDFs of meteorological elements, the PDF of the ampacity were forecasted. Based on the historical meteorological data, [10] utilized time series models to forecast the PDFs of meteorological elements, which then were used to forecast the probabilistic distribution of the ampacity.

The above studies on the probabilistic prediction of the ampacity did not use the QRNN model to consider the influence of historical meteorological data and historical ampacity data on the ampacity at predictive time period, which may reduce the accuracy of ampacity prediction results. Therefore, this paper proposes a QRNN-based method to improve the performance of the

*Corresponding author's e-mail: wangmx@sdu.edu.cn 
probabilistic prediction of the ampacity. The proposed method can forecast the ampacity directly by comprehensively modelling the effects of historical meteorological data and historical ampacity data on the ampacity at predictive time period, thus avoiding the prediction error caused by the inaccurate predictions of four meteorological elements.

The remainder of this paper is organized as follows. Section 2 introduces the ampacity calculation method. Section 3 presents the probabilistic prediction method of the ampacity. Section 4 analyses the prediction results and conclusions are drawn in section 5 .

\section{Ampacity calculation method}

According to the IEEE standard [11], the ampacity of an overhead conductor can be calculated by:

$$
I_{\max }=\left[\frac{q_{\mathrm{c}}+q_{\mathrm{r}}-q_{\mathrm{s}}}{R\left(T_{\max }\right)}\right]^{1 / 2},
$$

where $T_{\max }$ is the maximum allowable temperature of the conductor; $I_{\max }$ is the ampacity of the overhead conductor; $R\left(T_{\max }\right)$ is the resistance of the conductor at temperature $T_{\max } ; q_{\mathrm{c}}$ is the convection heat loss rate of the conductor; $q_{\mathrm{r}}$ is the radiated heat loss rate of the conductor; $q_{\mathrm{s}}$ is the heat gain rate from sun. The calculation formulas of $q_{\mathrm{s}}, q_{\mathrm{c}}$ and $q_{\mathrm{r}}$ are given as follows:

$$
\begin{gathered}
q_{\mathrm{s}}=a Q_{\mathrm{se}} \sin (\theta) A^{\prime}, \\
\left\{\begin{array}{l}
q_{\mathrm{c} 1}=\left[1.01+1.35\left(\frac{D_{0} \rho V_{\mathrm{w}}}{u}\right)^{0.52}\right] k k_{\mathrm{a}}\left(T_{\mathrm{s}}-T_{\mathrm{a}}\right) \\
q_{\mathrm{c} 2}=0.754\left(\frac{D_{0} \rho V_{\mathrm{w}}}{u}\right)^{0.6} k k_{\mathrm{a}}\left(T_{\mathrm{s}}-T_{\mathrm{a}}\right) \\
q_{\mathrm{c} 3}=3.645 \rho^{0.5} D_{0}^{0.75}\left(T_{\mathrm{s}}-T_{\mathrm{a}}\right)^{1.25} \\
q_{\mathrm{r}}\left(T_{\mathrm{s}}\right)=17.8 D_{0} e\left[\left(\frac{T_{\mathrm{s}}+273}{100}\right)^{4}-\left(\frac{T_{\mathrm{a}}+273}{100}\right)^{4}\right] .
\end{array}\right.
\end{gathered}
$$

In equation (2), $a$ is the solar absorptivity, $Q_{\mathrm{se}}$ is the solar radiation on the per unit square ground after elevation correction $\left(\mathrm{W} / \mathrm{m}^{2}\right), \theta$ is the effective angle of incidence of sun's rays $\left(^{\circ}\right), A^{\prime}$ is the projection area of conductor $\left(\mathrm{m}^{2} / \mathrm{m}\right)$; In equation (3), $D_{0}$ is the outside diameter of conductor $(\mathrm{m}), \rho$ is the air density $\left(\mathrm{kg} / \mathrm{m}^{3}\right), u$ is the air viscosity $(\mathrm{kg} / \mathrm{m}-\mathrm{s}), V_{\mathrm{w}}$ is the wind speed $(\mathrm{m} / \mathrm{s}), k$ is the coefficient of thermal conductivity of air, $k_{\mathrm{a}}$ is the wind direction factor. $T_{\mathrm{s}}$ is the conductor surface temperature. $q_{\mathrm{c} 1}$ and $q_{\mathrm{c} 2}$ are used to calculate the forced convection heat loss rates caused by the low wind speed and high wind speed, respectively. $q_{\mathrm{c} 3}$ is used to calculate the natural convective heat loss rates under zero wind speed. In equation (4), $e$ is the heat radiation coefficient of the conductor; $T_{\mathrm{a}}$ is the ambient temperature $\left({ }^{\circ} \mathrm{C}\right)$.

According to equation (1) to equation (4), after determining the location and type of an overhead line, the ampacity of the overhead line mainly varies with the variations of four meteorological elements around the overhead line (ambient temperature, wind speed, wind direction, and solar radiation).

\section{Probabilistic prediction method}

In this section, the QRNN model is applied for the probabilistic prediction of the ampacity at predictive time period. The description of the QRNN-based probabilistic prediction method is presented in subsection 3.1. The evaluation criteria for the probabilistic prediction model is provided in subsection 3.2.

\subsection{The QRNN-based probabilistic prediction method}

The flowchart of the QRNN-based probabilistic prediction method is shown in figure 1. As shown in figure 1, the historical meteorological data and ampacity data are inputted to the QRNN model to predict the quantiles of the ampacity at predictive time period. In this paper, the historical data of the predictive time period in the previous day combined with 1-hour historical data before the predictive time period are used as input data.

The QRNN model is a nonparametric and nonlinear model, which has been widely applied in the power load prediction [12]. Different from the traditional linear Quantile Regression (QR) model, the QRNN model can extract the nonlinear relationship between the output variables and the input variables. Moreover, the QRNN model can be used to implement probabilistic prediction, while the basic neural network model can only provide determined prediction. According to Taylor, the schematic diagram of a QRNN model with a single hidden layer is shown in figure 2 and the QRNN model can be expressed by:

$$
y(\tau)=g_{2}\left(\sum_{j=1}^{m} v_{j}(\tau) g_{1}\left(\sum_{i=1}^{n} w_{j i}(\tau) x_{i}\right)\right) .
$$




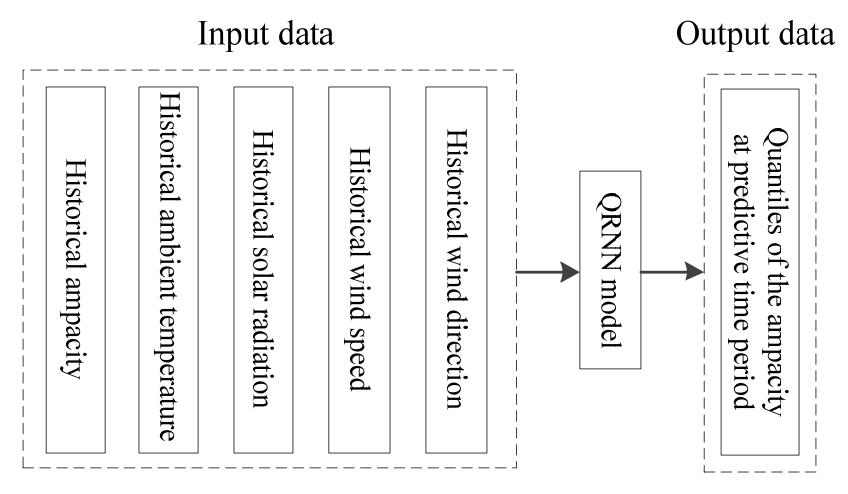

Figure 1. Flowchart of the prediction method.

In figure 2 and equation (5), $y(\tau)$ is the $\tau$ quantile of the ampacity prediction results; $n$ is the number of input variables; $x_{i}(i=1 \ldots n)$ are the $i^{\text {th }}$ input layer variable; $m$ is the number of hidden layer nodes; $w_{j i}(j=1 \ldots m)$ are the connection weight parameters between the $i^{\text {th }}$ parameter of the input layer and the $j^{\text {th }}$ hidden layer node; $v_{j}$ are the connection weight parameters between the $j^{\text {th }}$ hidden layer node and the output layer; $g_{1}(\cdot)$ and $g_{2}(\cdot)$ are activation functions, which are expressed by equation (6) and equation (7), respectively.

$$
\begin{array}{r}
g_{1}(t)=\frac{e^{t}-e^{-t}}{e^{t}+e^{-t}} \\
g_{2}(t)= \begin{cases}0, & t \leq 0 \\
t, & t>0\end{cases}
\end{array}
$$

The weight parameters in equation (5) can be estimated by optimizing the following objective function:

$$
F=\min \left(\sum_{r=1}^{z} \eta_{\tau}\left(Y_{r}-y_{r}(\tau)\right)+\lambda_{1} \sum_{j, i} w_{j i}^{2}(\tau)+\lambda_{2} \sum_{j} v_{j}^{2}(\tau)\right)
$$

where $z$ is the number of the sample; $\eta_{\tau}(\cdot)$ is the test function defined by equation (9); $Y_{r}(r=1 \ldots z)$ are the actual values of the ampacity; $\lambda_{1}$ and $\lambda_{2}$ are regularization parameters which constraint the complexity of the model.

$$
\eta_{\tau}(u)= \begin{cases}\tau \cdot u & u \geq 0 \\ (\tau-1) \cdot u & u<0\end{cases}
$$

After the parameter estimation, the input data and the estimated parameters are substituted to equation (5) to predict the quantiles of the ampacity at predictive time period.

\subsection{Evaluation criteria}

Quantile Score (QS) can comprehensively evaluate the reliability and sharpness of the performance of probabilistic prediction method [13]. The QS is defined as:

$$
\mathrm{QS}=\frac{1}{\operatorname{len}(Q)} \sum_{\tau \in Q} \eta_{\tau}(Y-y(\tau))
$$

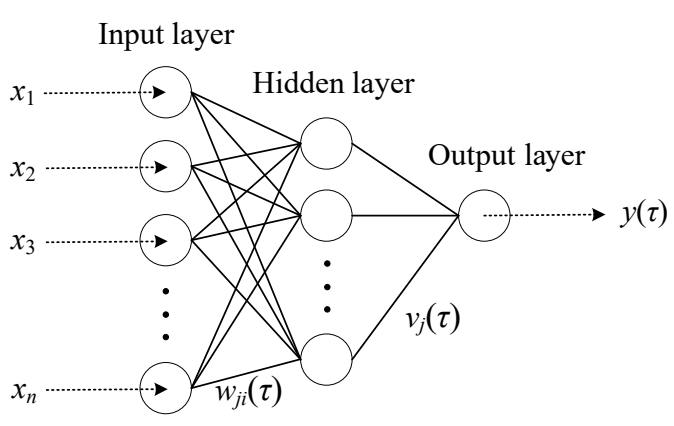

Figure 2. Schematic diagram for the QRNN model.

In equation (10), $Y$ is the actual value of the ampacity; $y(\tau)$ is the $\tau$ quantile of the ampacity prediction results; $Q$ is the quantile set of interest, $Q=\{0.01,0.02, \ldots, 1\} ; \eta_{\tau}(\cdot)$ is the test function defined by $(9)$; len $(Q)$ is the size of $Q$. As can be inferred from equation (10), the more accurate the prediction results are, the smaller the value of QS is. Therefore, the lower QS means better performance of the probabilistic prediction method.

\section{Case study}

In this case study, the meteorological data around an overhead transmission line from April 1st, 2018 to May 3rd, 2018 (33days) with 15-minute time resolution were collected. After that, the historical ampacity data for 33 days were calculated using equation (1) based on the collected meteorological data. The overhead conductor type is ACSR300. Its STR is 650 A which is calculated under given conservative weather conditions (air temperature $35^{\circ} \mathrm{C}$, wind speed $0.5 \mathrm{~m} / \mathrm{s}$ perpendicular to the conductor and the solar radiation is $800 \mathrm{~W} / \mathrm{m}^{2}$ ). The first 30-day historical data are used as the training dataset to estimate the parameters in the QRNN model, and the remainder 3-day data are used as the test dataset to validate the model.

\subsection{Probabilistic prediction results of the ampacity}

As described in Section 3, the historical meteorological data and historical ampacity data are taken as the input of the proposed QRNN-based method to predict the quantiles distribution of the ampacity directly (hereinafter called method I). We conduct the predictions for every 15 minutes up to 3 days. To verify the superiority of the proposed probabilistic prediction method, we also use the QRNN model to implement the probabilistic prediction of four meteorological elements firstly by only taking the historical meteorological data as input, and then calculate the quantiles distribution of the ampacity using equation (1) (hereinafter called method II). In addition, by using the same input data as method I, the linear QR model is also used to perform the probabilistic prediction of the ampacity directly (hereinafter called method III). The prediction results of method I, II and III are shown in figure 3. 


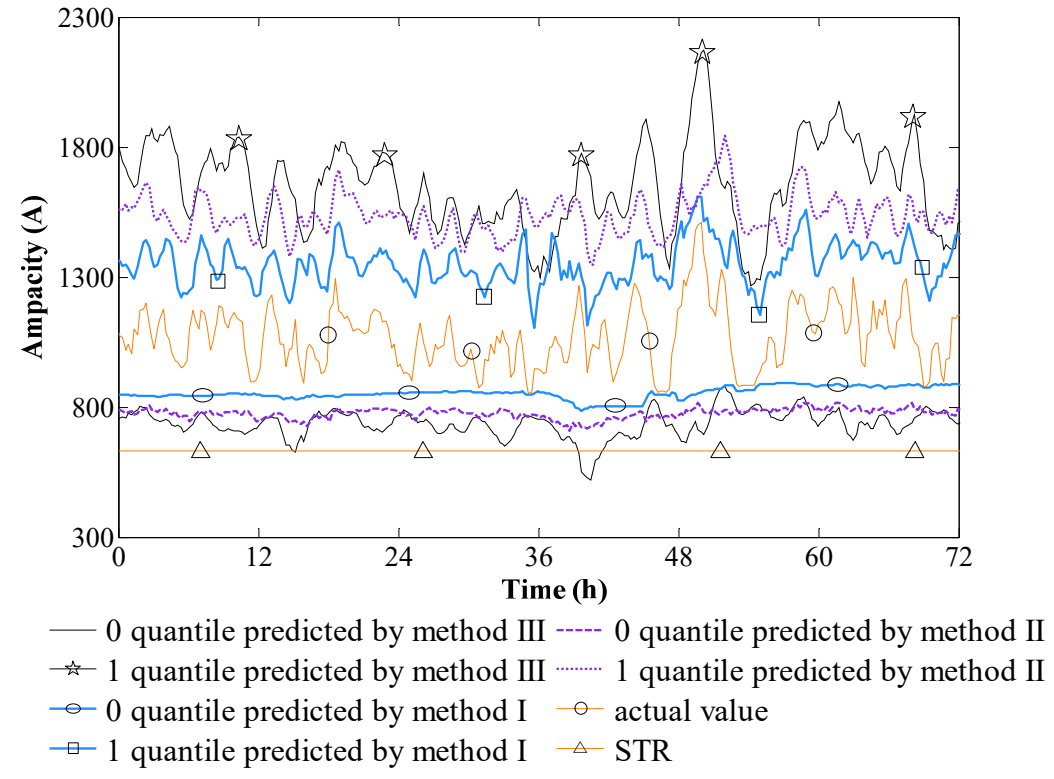

Figure 3. Actual ampacity, STR, prediction results of method I, II and III.

It can be seen from figure 3 that the prediction intervals of the method I, II and III can fully cover the actual ampacity, which indicates that three methods all have good performance on reliability. However, the prediction intervals of method I are the narrowest and the 0 quantiles predicted by method I are the largest. In this case, the prediction results of method I can guide operators to make more use of the transfer capacity of the overhead conductor.

\subsection{Evaluation of the probabilistic prediction results}

To accurately evaluate the performance of three probabilistic prediction methods, the QSs of the prediction results of three methods are calculated and shown in figure 4. The red dotted line in figure 4 represents the average QS.
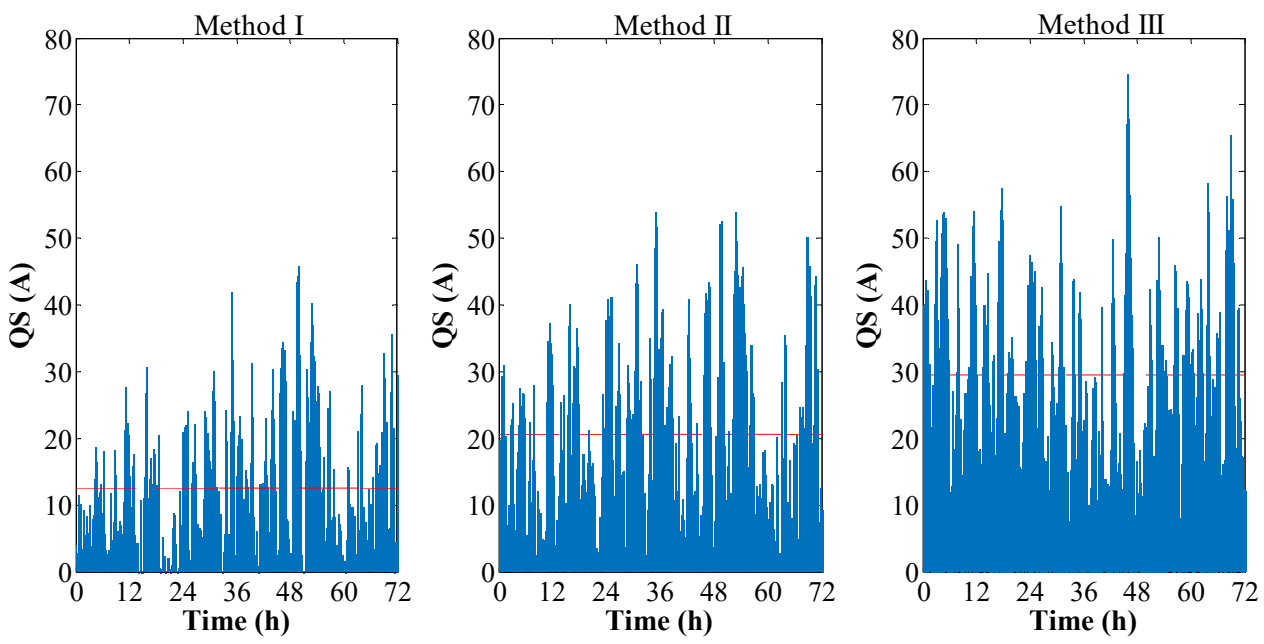

Figure 4. QSs of three probabilistic prediction methods.

As shown in figure 4, the QSs of the prediction results of method I are lower than that of the method II and method III in most times. The method I has the minimum average QS, followed by method II and method III. In this case, the QRNN-based method (method I) has a better performance on the probabilistic prediction of the ampacity than the linear QR model, and reduces the ampacity prediction error by avoiding the need of forecasting four meteorological elements.

\section{Conclusion}

In this paper, a QRNN-based method for the probabilistic prediction of the ampacity was proposed. The proposed method utilizes a nonlinear model and does not require the predictions of meteorological elements, thus improving the performance of the probabilistic prediction of the ampacity. Compared with the method using a linear QR model and the method requiring the 
meteorological elements predictions, the average QS of QRNN-based method is reduced by $57.37 \%$ and $39.12 \%$, respectively.

\section{Acknowledgments}

This work was supported in part by the Key Research and Development Plan of Shandong Province (2019GGX103044).

\section{References}

1. Alvarez, D.L., da Silva, F.F., Mombello, E.E., Bak, C.L., Rosero, J.A. (2018). Conductor temperature estimation and prediction at thermal transient state in dynamic line rating application. IEEE Transactions on Power Delivery, 33: 22362245.

2. Jiang, J., Liang, Y., Chen, C., Zheng, X., Chuang, C., Wang, C. (2018) On Dispatching Line Ampacities of Power Grids Using Weather-Based Conductor Temperature Forecasts. IEEE Transactions on Smart Grid, 9: 406-415.

3. Douglass, D., Chisholm, W., Davidson, G., Grant, I., Lindsey, K., Lancaster, M., Lawry, D., McCarthy, T., Nascimento, C., Pasha, M., Reding, J., Seppa, T., Toth, J., Waltz, P. (2016). RealTime overhead transmission-line monitoring for dynamic rating. IEEE Transactions on Power Delivery, 31: 921-927.

4. Bhattarai, B.P., Gentle, J.P., McJunkin, T., Hill, P.J., Myers, K.S., Abboud, A.W., Renwick, R., Hengst, D. (2018) Improvement of transmission line ampacity utilization by weather-based dynamic line rating. IEEE Transactions on Power Delivery, 33: 1853-1863.

5. Wallnerstrom, C.J., Huang, Y., Soder, L. (2015) Impact from dynamic line rating on wind power integration. IEEE Transactions on Smart Grid, 6: 343-350.

6. Kim, D., Kim, J. (2010) Prediction of transmission-line rating based on thermal overload probability using weather models. European Transactions on Electrical Power, 20: 534-544.

7. Kabovic, A.V., Kabovic, M.M., Bostjancic Rakas, S.V., Timcenko, V.V. (2018) Models for ShortTerm Forecasting of Parameters Used for Calculation of the Overhead Line Ampacity. In: 2018 26th Telecommunications Forum. Belgrade. pp. 1-4.

8. Konstantinou, T., Savvopoulos, N., Hatziargyriou, N. (2019) Probabilistic Ampacity Forecasting for Overhead Transmission Lines. In: 2019 International Conference on Smart Energy Systems and Technologies. Porto. pp. 1-6.

9. Ringelband, T., Schäfer, P., Moser, A. (2013) Probabilistic ampacity forecasting for overhead lines using weather forecast ensembles. Electrical Engineering, 95: 99-107.

10. Fan, F., Bell, K., Infield, D. (2017) Probabilistic real-time thermal rating forecasting for overhead lines by conditionally heteroscedastic autoregressive models. IEEE Transactions on Power Delivery, 32: 1881-1890.

11. (2013) IEEE Standard for Calculating the Current-Temperature Relationship of Bare Overhead Conductors. IEEE Std 738-2012. pp. 172.

12. He, Y., Qin, Y., Wang, S., Wang, X., Wang, C. (2019) Electricity consumption probability density forecasting method based on LASSOQuantile Regression Neural Network. Applied Energy, 233-234: 565-575.

13. Dupin, R., Kariniotakis, G., Michiorri, A. (2019) Overhead lines dynamic line rating based on probabilistic day-ahead forecasting and risk assessment. International Journal of Electrical Power and Energy Systems, 110: 565-578. 